\title{
二维疏水铜基纳米片的合成及在硫醚类化合物催化氧化中的应用
}

\author{
杨忠杰 ${ }^{\dagger, a, b}$ 张小飞卉 $a$ 施亚男 ${ }^{a, b}$ 隆昶 ${ }^{c}$ 张涁影 ${ }^{a, d}$ \\ 闵书豪 $a, d$ 常琳 $a, b$ 唐智勇*, $a, b$ \\ $\left({ }^{a}\right.$ 国家纳米科学中心 中国科学院纳米系统与多级次制造重点实验室 北京 100190) \\ ( ${ }^{b}$ 中国科学院大学 纳米科学与技术学院 北京 100049) \\ ( ${ }^{c}$ 哈尔滨工业大学 材料学院 哈尔滨 150001) \\ $\left({ }^{d}\right.$ 中国科学院大学中丹学院 北京 100049)
}

\begin{abstract}
摘要 二维纳米材料因其具有独特的物理化学性质得到了广泛的研究与关注. 然而, 自下而上制备稳定的二维纳米材 料仍然面临巨大挑战. 本工作以 1,4-苯二硫醇为有机配体和 $\mathrm{Cu}^{+}$为金属前驱体，通过配位相互作用自组装构筑了一种新 型的二维金属有机配合物纳米片(Cu-BDT). Cu-BDT 纳米片的形貌和结构被多种技术详细表征，包括粉末 X 射线衍射 (P-XRD)、傅里叶变换红外光谱(FT-IR)、激光拉曼光谱(Raman)、扫描电子显微镜(SEM)、透射电子显微镜(TEM)、原 子力显微镜(AFM)、X 射线光电子能谱(XPS)、电感耦合等离子体发射光谱仪(ICP-OES)和接触角测试. 催化实验证实, $\mathrm{Cu}-\mathrm{BDT}$ 纳米片表面暴露充分的活性位点和疏水特性有利于硫醚类化合物氧化反应的进行, 可高效转化为亚砜类产物. 关键词 金属有机配合物; 二维纳米片; 疏水性; 硫醚类化合物氧化
\end{abstract}

\section{Synthesis of Two-dimensional Hydrophobic Copper-based Nanosheets and Their Application in Catalytic Oxidation of Sulfides}

\author{
Yang, Zhongjie $\mathrm{e}^{\dagger, a, b} \quad$ Zhang, Xiaofei ${ }^{\dagger+a} \quad$ Shi, Yanan $^{a, b} \quad$ Long, Chang $^{c} \quad$ Zhang, Binhao ${ }^{a, d}$ \\ Yan, Shuhao ${ }^{a, d} \quad$ Chang, Lin ${ }^{a, b} \quad$ Tang, Zhiyong*,a,b \\ $\left({ }^{a}\right.$ CAS Key Laboratory of Nanosystem and Hierarchical Fabrication, CAS Center for Excellence in Nanoscience, \\ National Center for Nanoscience and Technology, Beijing 100190, China) \\ ( ${ }^{b}$ School of Nanoscience and Technology, University of Chinese Academy of Sciences, Beijing 100049, China) \\ ( ${ }^{c}$ School of Materials Science and Engineering, Harbin Institute of Technology, Harbin 150080, China) \\ ( ${ }^{d}$ Sino-Danish Center for Education and Research, Sino-Danish College, University of Chinese Academy of Sciences, \\ Beijing 100049, China)
}

\begin{abstract}
Two-dimensional nanomaterials have received extensive attention because of their unique physicochemical properties. However, bottom-up synthesis of two-dimensional (2D) stable nanomaterials still remains great challenge. In this work, a novel 2D metal-organic (Cu-BDT) nanosheet is constructed at room temperature by coordinative self-assembly, namely, using monovalent copper ion as the metal precursor and 1,4-benzenedithiol as organic ligand. As-synthesized $\mathrm{Cu}$-BDT nanosheets are fully characterized by various techniques including powder-diffraction of X-rays (P-XRD), Fourier transform infrared spectrometer (FT-IR), Raman spectra (Raman), scanning electron microscopy (SEM), transmission electron microscope (TEM), atomic force microscope (AFM), X-ray photoelectron spectroscopy (XPS), inductively coupled plasma optical emission spectrometer (ICP-OES) and contact angle test. The catalytic result verifies that the Cu-BDT nanosheet surfaces possess abundant active sites and good hydrophobicity, which facilitate oxidation of sulfides into sulfoxide compounds.

Keywords metal organic coordination complex; two-dimensional nanosheet; hydrophobicity; sulfides oxidation
\end{abstract}

\footnotetext{
* E-mail: zytang@nanoctr.cn

$\dagger$ 共同第一作者(These authors contributed equally to this work).

Received May 12, 2020; published July 9, 2020.

Supporting information for this article is available free of charge via the Internet at http://sioc-journal.cn.

Project supported by the Strategic Priority Research Program of Chinese Academy of Sciences (No. XDB36000000), National Key Basic Research Program of China (No. 2016YFA0200700), National Natural Science Foundation of China (Nos. 21890381, 21721002), Frontier Science Key Project of Chinese Academy of Sciences (No. QYZDJ-SSW-SLH038), and K.C.Wong Education Foundation.

项目受中国科学院战略重点研究计划(No. XDB36000000)、国家重点基础研究计划(No. 2016YFA0200700)、国家自然科学基金(Nos. 21890381, 21721002)、中国科学院前沿科学重点项目(No. QYZDJ-SSW-SLH038)和王宽诚教育基金会资助.
} 


\section{1 引言}

具有原子级厚度的二维材料在能源储存 ${ }^{[1-5] \text { 、分离 }}{ }^{[6]}$ 和催化 ${ }^{[7-12]}$ 等方面得到了广泛研究. 二维材料常见的合 成方法包括自上而下 ${ }^{[13-15]}$ 和自下而上 ${ }^{[16]}$ 两种. 总体来 说, 无机二维材料常用的制备方法为自上而下合成, 即 通过机械力将块体结构剥离成层状结构, 但这种方法不 利于制备高质量和大批量的纳米材料 ${ }^{[17,18]}$. 对于合成二 维金属有机配合物材料而言 ${ }^{[19]}$, 自下而上合成方法不 仅可以调控金属前驱体和有机配体，还可以显著地提高 二维纳米材料的合成产率. 在金属有机配合物体系中, 多颈基配体很容易与后过渡金属离子配位，在界面生长 即可获得二维纳米片 ${ }^{[20-25]}$, 例如朱道本等 ${ }^{[26,27]}$ 使用 $\mathrm{Ag}^{+}$ 和 $\mathrm{Cu}^{+}$离子与六颈基苯在界面自组装形成了稳定的纳米 片层材料, 但该方法产率仍然较低. 相反, 将两种前驱 体直接在溶液中混合, 得到产物往往是大量块体材料. 这是因为颈基与金属的配位速度要明显快于羧基和吡 啶与金属的配位 ${ }^{[28-30], ~}$ 常见的一锅法很难保证合成高纯 度的片层材料. 就颈基与金属配位产物具体而言, 迄今 尚未见文献报道一锅法合成大量的纳米片层材料.

含硫化合物的氧化是工业脱硫的重要反应, 不仅可 以将原油中的有机硫化物去除, 减少对环境的污染, 且 氧化得到的亚砜与砜具有重要的化工价值 ${ }^{[31]}$. 常见的 亚砜合成方法是将硫醚键氧化, 而在氧化过程中, 过氧 化后的产物是更加廉价的砜, 因此, 如何调控硫醚氧化 得到亚砜是合成过程中的关键. 目前常用的催化剂主要 包含层状氢氧化物 ${ }^{[32]}(\mathrm{LDH})$ 和块体材料包覆的杂多 酸 ${ }^{[33]}(\mathrm{POM})$, 它们不仅合成繁琐且常常会将硫醚过度氧
化. 王训等 ${ }^{[34]}$ 报道了长烷基链修饰的 POM 的催化性能 要优于短链修饰的性能, 主要归因于硫醚具有强的疏水 性，有助于底物的吸附，而产物亚砜具有弱的疏水性， 有助于脱附. 同年 ${ }^{[35]}$, 研究发现磁性 $\mathrm{Fe}_{3} \mathrm{O}_{4}$ 纳米颗粒负 载在经过疏水处理的 POM 表面, 可以迅速将硫醚氧化 成砜，主要归因于硫醚具有弱的极性，疏水有助于底物 的吸附, 而产物具有强的极性, 有助于产物的脱附. 硫 醚类化合物大多数具有弱的极性和强的疏水性, 而氧化 后得到的亚砜和砜具有强的极性和弱的疏水性, 因此通 过改善催化剂的疏水性可以促进反应的转化率和选择. 目前未有工作报道二维材料用于硫醚类化合物氧化.

综上所述, 本研究通过铜离子与䘪基在乙腈溶液中 自组装形成大量的厚度小于 $5 \mathrm{~nm}$ 的纳米片, 根据材料 的疏水特性, 以双氧水 $\left(\mathrm{H}_{2} \mathrm{O}_{2}\right)$ 为氧化剂, 在最佳条件下 表现出优异的性能. 不同于已经报道的块体催化剂, 超 薄铜基纳米片催化剂能够充分暴露活性位点, 更有利反 应底物和产物的传输, 表现出优异的催化性能.

\section{2 结果与讨论}

\section{1 材料的表征}

将合成得到的 $\mathrm{Cu}-\mathrm{BDT}$ 纳米片进行了详细的表征, 包括粉末 $\mathrm{X}$ 射线衍射(P-XRD)、傅里叶变换红外光谱 (FT-IR)、激光拉曼光谱(Raman)、扫描电子显微镜(SEM)、 透射电子显微镜(TEM)、原子力显微镜(AFM)、 $X$ 射线 光电子能谱 $(X P S)$ 、电感耦合等离子体发射光谱仪 (ICP-OES)和接触角测试表征，具体结果分别阐述如下. $\mathrm{Cu}-\mathrm{BDT}$ 的制备过程如图 1a, 将氯化亚铜 $(\mathrm{CuCl})$ 和 1,4-

Cu-BDT 的制备过程如图 1a, 将氯化亚制(CuCl)和 $1,4-$
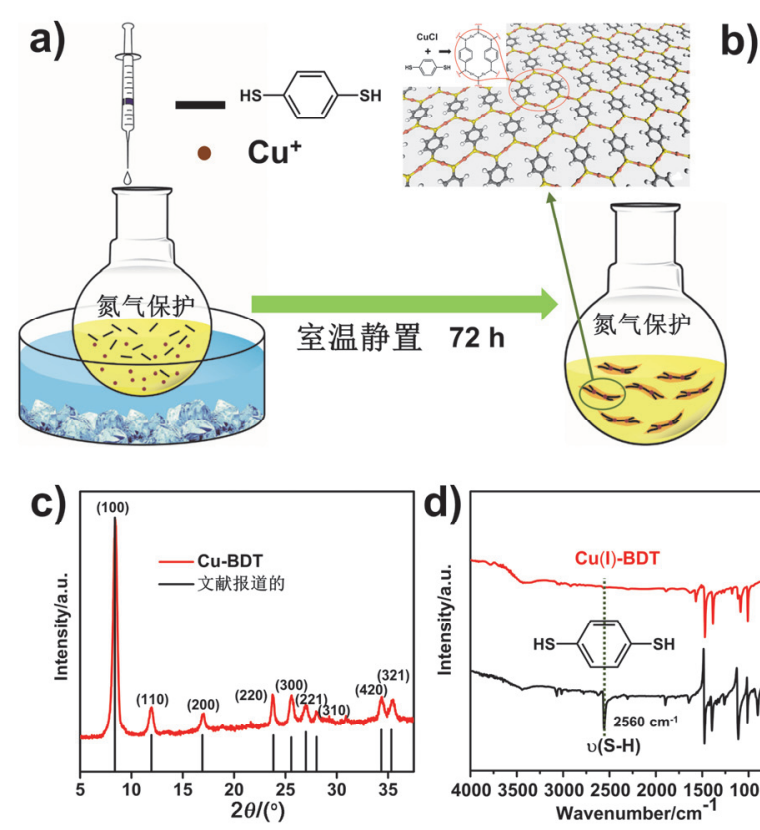

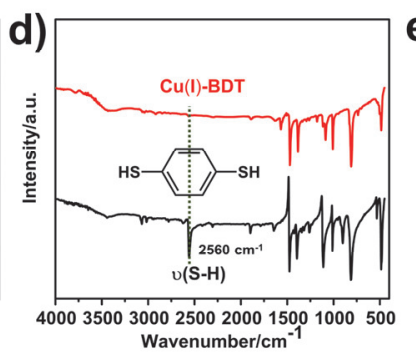

图 1 (a) $\mathrm{Cu}-\mathrm{BDT}$ 合成及结构示意图. (b) $\mathrm{Cu}-\mathrm{BDT}$ 固体粉末及在乙醇溶液中形成的丁达尔散射现象. (c)文献报道过的及本文合成的 $\mathrm{Cu}-\mathrm{BDT}$ 的 XRD. BDT 及 Cu-BDT 的(d) FT-IR 谱图和(e) Raman 谱图.

Figure 1 (a) Scheme of the formation and structure of Cu-BDT. (b) Cu-BDT solid powder image and Tyndall light scattering of Cu-BDT nanosheets in ethanol solution. (c) The XRD patterns of Cu-BDT reported in the literature and synthesized in this paper. (d) FT-IR and (e) Raman spectra of BDT and Cu-BDT. 
苯二硫醇(BDT)分别溶于超干乙腈溶液中, 在冰浴条件 下慢慢地将含有 $\mathrm{BDT}$ 的溶液滴加到 $\mathrm{N}_{2}$ 保护的 $\mathrm{CuCl}$ 溶 液中, 混合溶液恢复至室温后静置 $72 \mathrm{~h}$, 通过 $\mathrm{Cu}^{+}$与硫 醇的配位作用形成大量的黄色絮状沉淀(Cu-BDT). 离 心洗涤后得到的黄色固体如图 $1 \mathrm{~b}$ 所示, 根据二维超薄 材料的自身特征, 将少量的固体分散在乙醇溶液中, 静 置几个月后仍可以观察到非常明显的丁达尔散射现象, 这说明了合成的黄色固体具有类似的特征. 为了说明合 成的 $\mathrm{Cu}-\mathrm{BDT}$ 形貌特征, SEM 表征如图 2a 所示, 可以观 察到大量的二维片状材料, 且所得到的纳米片纵向大小 不均一, 表面平整干净. 如图 2b 所示, TEM 表征进一步 说明了 $\mathrm{Cu}-\mathrm{BDT}$ 为二维纳米片. 为了确定合成的 $\mathrm{Cu}-\mathrm{BDT}$ 纳米片中各种元素的分布情况, 通过 TEM-Mapping 表征, 如图 2c 所示, $\mathrm{Cu}-\mathrm{BDT}$ 中 $\mathrm{Cu} 、 \mathrm{~S}$ 和 $\mathrm{C}$ 元素均匀分布片状结构中, 没有观察到 $\mathrm{Cl}$ 元素的存 在, 这说明了 $\mathrm{Cu}-\mathrm{BDT}$ 是由 $\mathrm{CuCl}$ 脱氯和对苯二硫醇脱 氢进行配位的. 通过以上表征, 可以基本确定 $\mathrm{Cu}-\mathrm{BDT}$ 的形貌特征. AFM 表征了 $\mathrm{Cu}-\mathrm{BDT}$ 的厚度, 图 $2 \mathrm{~d}$ 所示, 所得到纳米片的厚度小于 $5 \mathrm{~nm}$, 在纳米尺度下, 可以定 义为二维超薄纳米片 ${ }^{[17]}$.

P-XRD 数据分析了 $\mathrm{Cu}-\mathrm{BDT}$ 的结构信息, 可以看出 合成的纳米片具有结晶性, 而不是简单线性聚合物, 如 图 1c 所示, 最强的衍射峰出现在 $2 \theta=8.5^{\circ}$ 处, 说明了 $\mathrm{Cu}-\mathrm{BDT}$ 沿着(100)晶面生长, 有利于纳米片的形成, 其 他的衍射峰位置与 Prakash 等 ${ }^{[36]}$ 报道的一致. 由于目前
尚未长出 $\mathrm{Cu}-\mathrm{BDT}$ 单晶，因此根据文中现有的表征，提 供了如图 $1 \mathrm{a}$ 中插图的可能结构示意图. 为了确定 $\mathrm{Cu}-\mathrm{BDT}$ 的配位情况, FT-IR 表征如图 1d 所示, BDT 中巯 基的吸收振动峰的位置是 $2560 \mathrm{~cm}^{-1}$, 而在 $\mathrm{Cu}-\mathrm{BDT}$ 中, 颈基的振动吸收峰完全消失，这表明了配体中的巯基完 全与铜离子进行了配位. 为了进一步说明 $\mathrm{Cu}-\mathrm{BDT}$ 中化 学键的情况, Raman 表征如图 1e 所示, Cu-BDT 中颈基的 振动峰在 $2558 \mathrm{~cm}^{-1}$ 消失了, 且在低于 $600 \mathrm{~cm}^{-1}$ 出现了 一系列新的振动峰, 根据郑南峰等 ${ }^{[37]}$ 的报道, 新的吸收 振动正是金属与颈基中硫形成的金属-硫键 $(\mathrm{Cu}-\mathrm{S}$ 键), 这也证明了颈基与铜离子进行了配位. XPS 分析了 $\mathrm{Cu}-\mathrm{BDT}$ 中各元素的价态情况, 如图 3a 所示, 通过判断 $\mathrm{Cu} 2 \mathrm{p}_{3 / 2}$ 的位置, 发现峰位置是 $933.2 \mathrm{eV}$, 这说明了 $\mathrm{Cu}-\mathrm{BDT}$ 中的铜是 $\mathrm{Cu}(\mathrm{I})$ 或者 $\mathrm{Cu}(0)$, 且这个峰位置与 $\mathrm{CuCl}$ (XPS 峰位置 $933.4 \mathrm{eV})^{[38]}$ 的峰位置匹配, 另外 $\mathrm{Cu}(\mathrm{I})-\mathrm{S}$ 键的峰位置在 $932.3 \sim 933.6 \mathrm{eV}^{[39]}$, 这些数据说 明了 $\mathrm{Cu}-\mathrm{BDT}$ 中的铜是一价或零价且也进一步说明了铜 离子与颈基配位的事实. 为了进一步说明 $\mathrm{Cu}-\mathrm{BDT}$ 中铜 的价态, 可以通过区别 $\mathrm{Cu}(\mathrm{I})$ 和 $\mathrm{Cu}(\mathrm{II})$ 卫星峰(940 945 $\mathrm{eV}^{[40]}$ 是 $\mathrm{Cu}(\mathrm{II})$ 的卫星峰), 从图 $3 \mathrm{a}$ 中可以看出该峰已经 消失, 说明了 $\mathrm{Cu}-\mathrm{BDT}$ 中铜的价态为一价. 另如图 $3 \mathrm{~b}$ 所 示, $\mathrm{Cu}$ 的俄歇电子谱线出现在 $570.9 \mathrm{eV}\left(\mathrm{Cu}^{+}\right)$而不是 $568.1 \mathrm{eV}\left(\mathrm{Cu}^{0}\right)$, 这与文献报道相同，通过上述分析确认 了 $\mathrm{Cu}-\mathrm{BDT}$ 中的铜仍是一价. 如图 3c 所示, $\mathrm{S}$ 元素的 $\mathrm{S}$ $2 p_{1 / 2}$ 和 $S 2 p_{3 / 2}$ 的位置分别在 $165.1 \mathrm{eV}$ 和 $164.1 \mathrm{eV}$ 处. 在
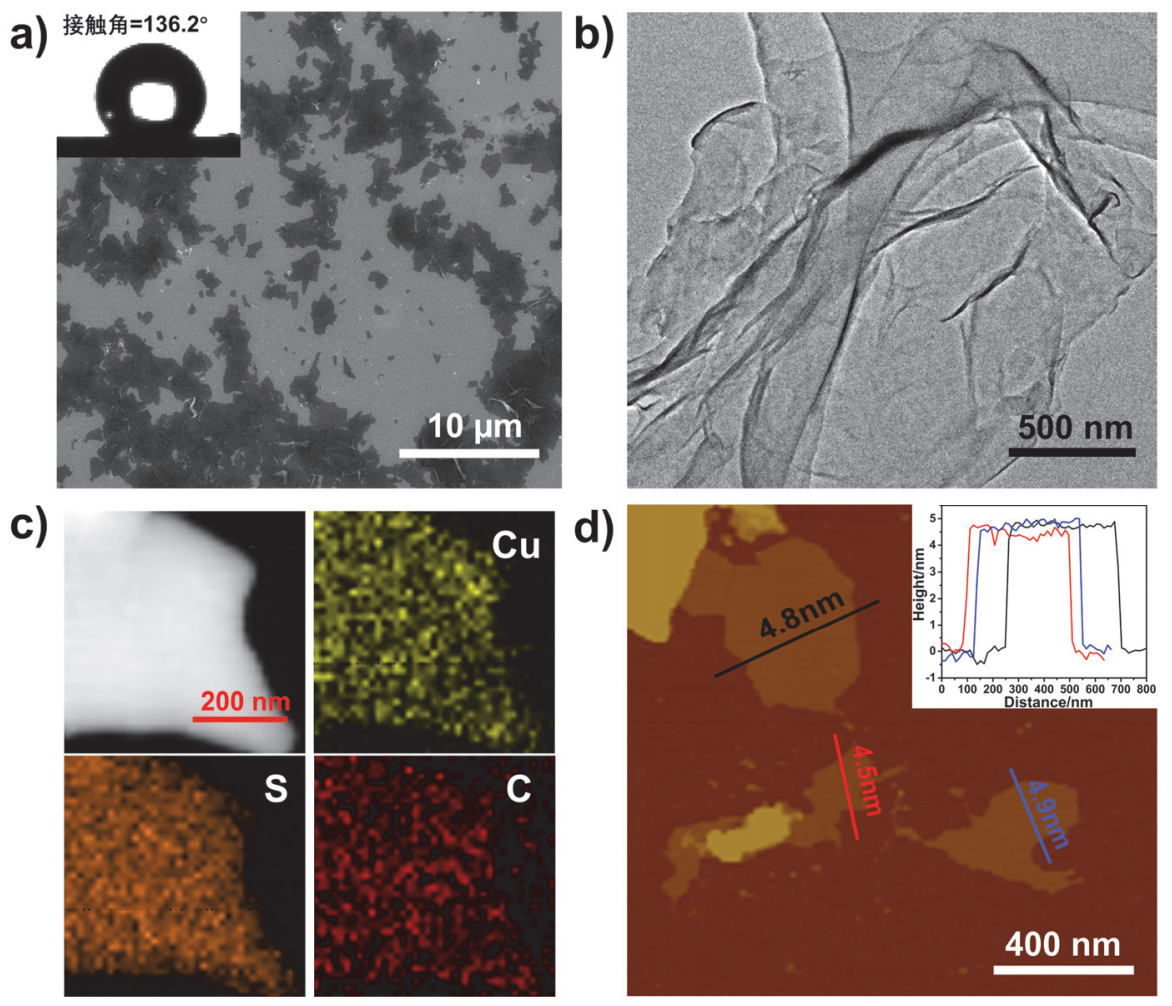

图 $2 \mathrm{Cu}-\mathrm{BDT}$ 纳米片的(a)扫描电镜和接触角测试图和(b)透射电镜图. (c) $\mathrm{Cu} 、 \mathrm{~S} 、 \mathrm{C}$ 元素分布图. (d) 原子力显微镜图和厚度分布情况.

Figure 2 The (a) SEM and (b) TEM images of Cu-BDT nanosheets, the inset shows Cu-BDT nanosheets are hydrophobic. (c) Elemental mapping images of $\mathrm{Cu}$-BDT nanosheets. (d) AFM image and corresponding height profile of $\mathrm{Cu}-\mathrm{BDT}$ nanosheets. 

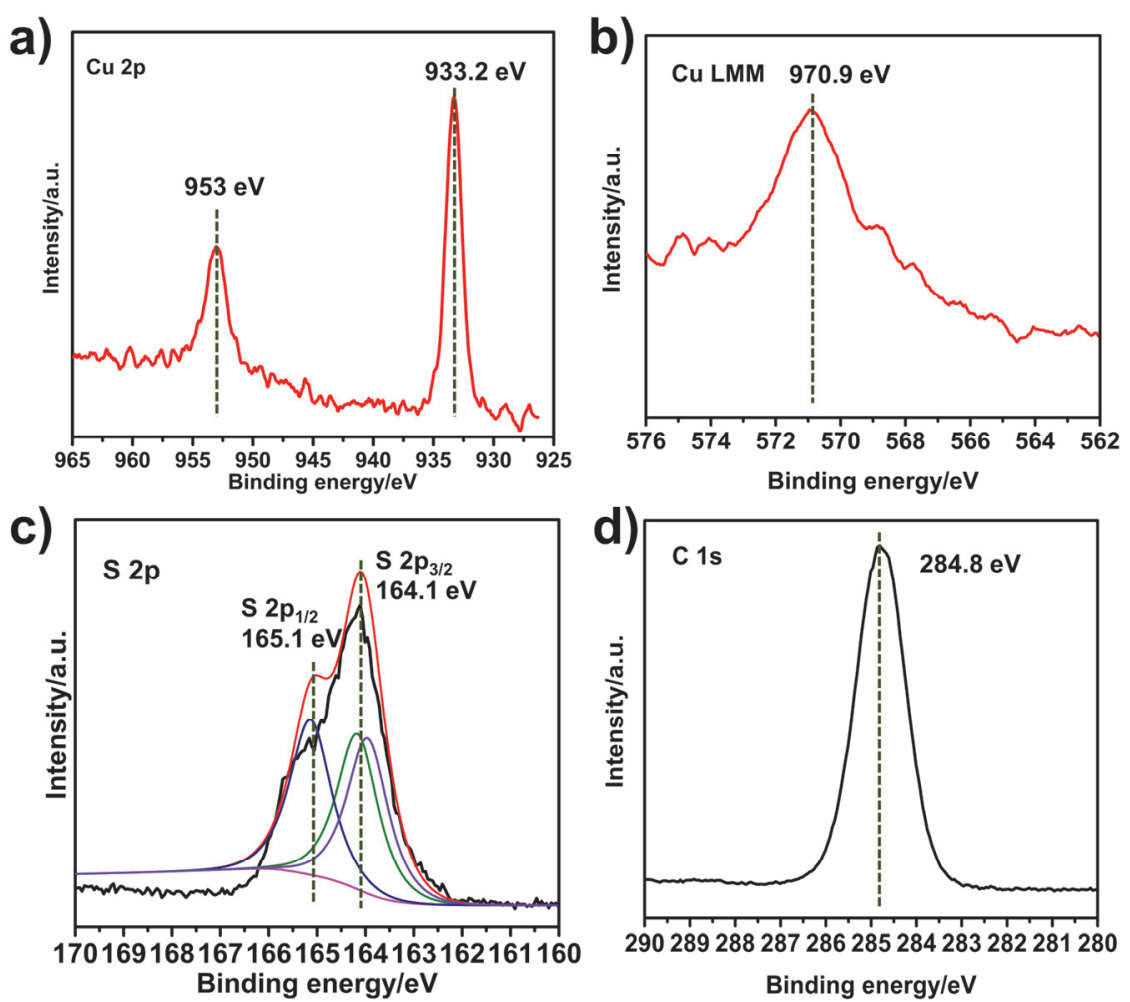

图 3 (a) Cu-BDT 纳米片中铜 $2 p$ 的 XPS 谱图. (b)铜的俄歇谱线. 硫 $2 \mathrm{p}$ 的 XPS 谱图(c)和碳 $1 \mathrm{~s}$ 的 XPS 谱图(d).

Figure 3 (a) Cu 2p XPS. (b) Cu LMM Auger. High-resolution S 2p XPS (c) and C 1s XPS spectra (d) of Cu-BDT.

$163.5 \mathrm{eV}$ 位置处对应的是 $\mathrm{S}-\mathrm{H}$ 键 ${ }^{[35]}$, 而在图中该位置 的峰并未出现, 这说明了 BDT 的袏基与铜离子已经发 生完全配位. $\mathrm{S}-\mathrm{S}$ 键中硫的 $2 \mathrm{p}_{3 / 2}$ 在 $164.4 \mathrm{eV}$, 而在谱图 中并未观察到, 这也说明了颈基与铜离子配位而并未形 成 $\mathrm{S}-\mathrm{S}$ 键 ${ }^{[41]}$. 从图 $3 \mathrm{c}$ 可以看出主要的两个峰是 164.1 $\mathrm{eV}$ 和 $165.1 \mathrm{eV}$, 这两个峰对应的是 $\mathrm{Cu}-\mathrm{S}$ 键 ${ }^{[2]}$. 图 3d 是 $\mathrm{Cu}-\mathrm{BDT}$ 中碳元素的 XPS 分析, 从图中可以观察到峰 位置在 $284.5 \mathrm{eV}$ 是典型的 $\mathrm{sp}^{2}$ 形式的碳 ${ }^{[43]}$, 即 BDT 中苯 环碳形式, 且没有 $\mathrm{S}=\mathrm{C}$ 键存在. 以上分析说明了 $\mathrm{Cu}-\mathrm{BDT}$ 中的铜元素是一价, $\mathrm{S}-\mathrm{H}$ 官能团全部与铜离子 配位且不存在 S-S 键, 从碳元素的 XPS 图谱可以看出 没有 $\mathrm{S}=\mathrm{C}$ 键出现. 如图 $\mathrm{S} 5, \mathrm{Cu}-\mathrm{BDT}$ 的热重数据分析表 明, 在 $0 \sim 400{ }^{\circ} \mathrm{C}$ 之间, 约有 $2.5 \%$ 的质量损失, 分析可 知为材料中残留溶剂的挥发所致, 直到 $450{ }^{\circ} \mathrm{C}$ 左右时, $\mathrm{Cu}-\mathrm{BDT}$ 发生明显的分解, 这表明了 $\mathrm{Cu}-\mathrm{BDT}$ 在氮气条 件下的热稳定性非常好. 为了进一步了解 Cu-BDT 的物 理化学性质, 测试了材料的接触角, 如图 2a 中的插图所 示, 得到接触角为 $136.2^{\circ}$, 根据亲疏水的定义, 所合成 的 $\mathrm{Cu}-\mathrm{BDT}$ 二维纳米片呈疏水特性, 这一性质为我们探 索该材料在催化方面的应用提供了重要线索.

综上所述，基于自下而上的合成方法，用廉价的 $\mathrm{CuCl}$ 为金属前驱体，1,4-苯二硫醇为有机配体，用简易 的合成方法得到了大量的黄色固体, 根据扫描电镜和透 射电镜的表征, 可以观察到所合成的 $\mathrm{Cu}-\mathrm{BDT}$ 为二维层 状形貌，原子力显微镜表征发现 $\mathrm{Cu}-\mathrm{BDT}$ 的厚度小于 5 $\mathrm{nm}, \mathrm{FT}-\mathrm{IR}$ 中 $\mathrm{Cu}-\mathrm{BDT}$ 的颈基完全消失，说明颈基全部 与 $\mathrm{Cu}^{+}$进行配位, 同时, 常温固体 Raman 图中也观察到 颈基消失, 且在低于 $600 \mathrm{~cm}^{-1}$ 处出现了一系列新的振动 峰, 这些峰是 $\mathrm{Cu}-\mathrm{S}$ 键的吸收振动. 为了确定 $\mathrm{Cu}-\mathrm{BDT}$ 中铜、硫和碳元素的化学状态, XPS 表征发现 Cu-BDT 中铜的价态仍然保持一价, 硫与碳元素的 XPS 分析说 明了 $\mathrm{Cu}-\mathrm{BDT}$ 中 $\mathrm{S}-\mathrm{H}$ 键完全配位且不存在 $\mathrm{S}-\mathrm{S}$ 键和 $\mathrm{S}=\mathrm{C}$ 键. 为了进一步分析 $\mathrm{Cu}-\mathrm{BDT}$ 的物理化学性质, 接 触角测试表明了 $\mathrm{Cu}-\mathrm{BDT}$ 具有疏水特性.

\section{2 硫醚类化合物的氧化}

\subsection{1 催化反应的条件优化}

铜基催化剂催化氧化反应已经得到了广泛研 究 ${ }^{[44-46]}$, 对于 $\mathrm{Cu}-\mathrm{BDT}$ 在催化硫醚氧化中的探究却还很 少. 为了探索影响反应的条件, 我们控制底物的量、催 化剂的量及反应温度, 对三个底物分别加入不同物质的 量的双氧水, 如图 $4 \mathrm{a} \sim 4 \mathrm{c}$ 所示, 当双氧水/底物的物质 的量比为 1 时, 茴香硫醚、4-甲基茴香硫醚和 4-氯茴香 硫醚的转化率都低于 $20 \%$, 其选择性接近 $100 \%$, 随着 双氧水与底物的比值增加, 转化率逐渐上升, 当双氧水/ 底物为 6 时，三个底物的转化率和选择性都接近 $100 \%$, 随着双氧水与底物比例的继续升高，底物达到完全转 化, 且选择性保持 $90 \%$, 整体而言, 当比值大于 6 后, 转 化率和选择性几乎保持不变, 这也有利于节省双氧水的 用量, 图 $4 \mathrm{a} \sim 4 \mathrm{c}$ 也表明了底物上吸电子基团和给电子 

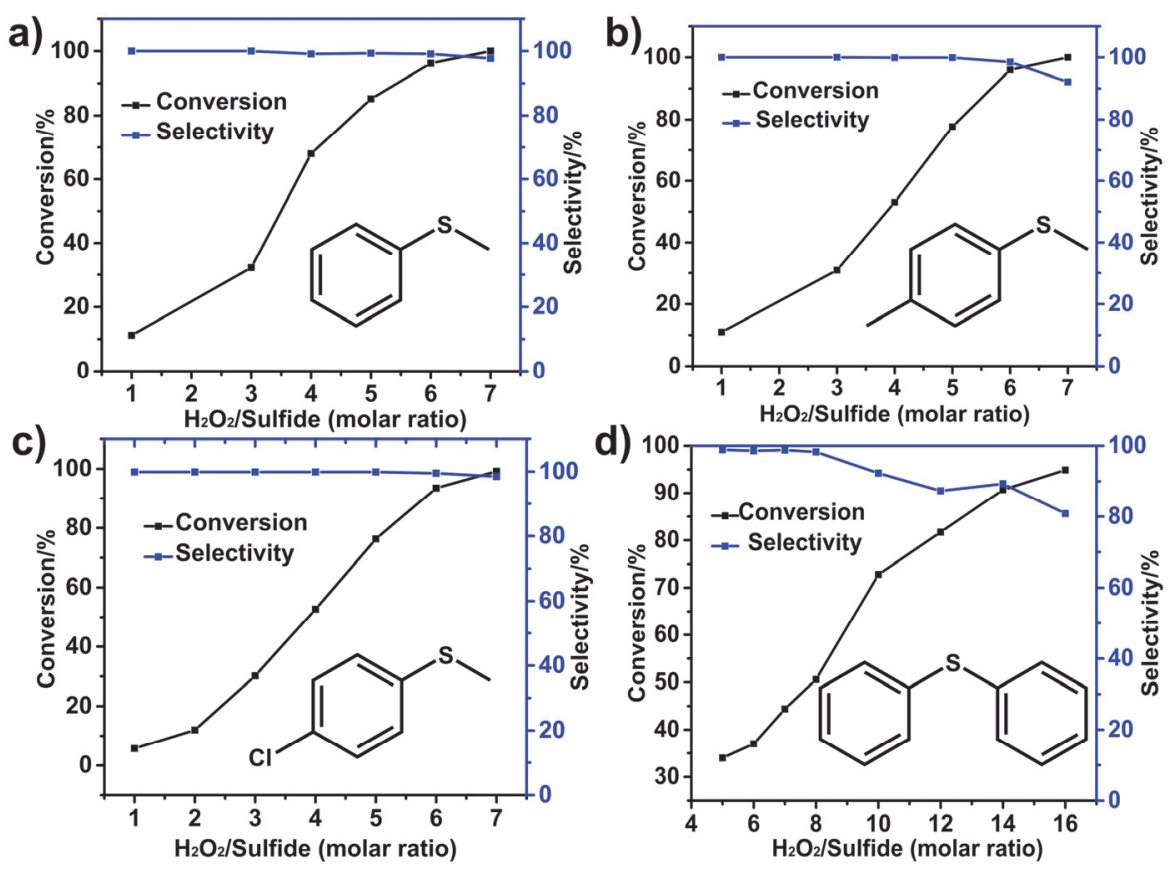

图 4 氧化剂的量对(a)茴香硫醚, (b) 4-甲基茴香硫醚, (c) 4-氯茴香硫醚和(d)二苯硫醚氧化的影响.

Figure 4 Effect of oxidant amount on thioanisole (a), methy $p$-tolylsulfide (b), 4-chlorothioanisol (c) and diphenyl sulfide (d) oxidation reaction.

基团对催化的反应活性没有明显的影响. 在探索双氧 水/底物之比对二苯硫醚氧化的影响时, 我们发现在低 比值时，转化率低于 $60 \%$, 如图 $4 \mathrm{~d}$ 所示, 当比值大于 10 时, 转化率达到 $70 \%$, 选择性大于 $80 \%$, 随着比值的增 加, 在比值为 14 时, 转化率接近 $100 \%$ 且选择性高达 $90 \%$, 由于二苯硫醚两端为苯环链接, 其中硫原子的电 子云密度高于其他底物中硫原子的电子云密度, 这导致 在小比值下无法很好地转化, 另外, 二苯硫醚具有强的 亲电效应, 弱的亲核作用, 而随着硫醚类化合物的亲电 性增加, 相应的氧化速率会减小. 基于最佳的优化条件 及 $\mathrm{Cu}$-BDT 的 ICP-OES 数据(如表 S1), 如表 1 所示, 相 比于已报道的文献(如表 S4 所示), Cu-BDT 对各类底物 的催化表现出优异的转换数 (TON), 这也说明了 $\mathrm{Cu}-\mathrm{BDT}$ 的超薄和疏水特性是有利于催化硫醚类化合物 氧化.

前面我们通过控制底物的量、催化剂的量及反应温 度, 探究了双氧水/底物比值对茴香硫醚、4-甲基茴香硫 醚、4-氯茴香硫醚和二苯硫醚氧化影响, 众所周知, 氧 化还原反应发生的速率非常快, 因此时间对反应的转化 率和选择性也有非常明显的影响. 我们选择了 4-甲基茴 香硫醚作为特征研究底物, 首先将 $5 \mathrm{mg} \mathrm{Cu}$-BDT 溶于 5 $\mathrm{mL}$ 异丙醇中, 然后加入 $1 \mathrm{mmol}$ 的 4-甲基茴香硫醚, 超 声至催化剂均匀分散后, 将该反应液移至两口玻璃反应 器中, 加入 $6 \mathrm{mmol}$ 的双氧水, 氮气置换三次后, $50{ }^{\circ} \mathrm{C}$ 摚拌一定时间. 如图 5a 所示, 在前 $1 \mathrm{~h}$ 内, 每隔 $10 \mathrm{~min}$ 取 1 次反应液, 随着时间的延长, 底物的转化率逐渐升 高, 且选择性保持不变, 当反应进行了 $5 \mathrm{~h}$ 后, 整个反应 体系的转化率几乎接近 $100 \%$, 选择性接近 $100 \%$, 随着
表 1 硫醚类化合物的氧化

Table 1 Sulfoxidation of sulfides ${ }^{a}$

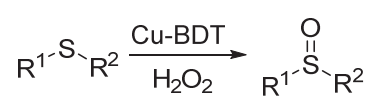

\begin{tabular}{|c|c|c|c|c|}
\hline 序号 & 底物 & 转化率 ${ }^{b} / \%$ & 选择性 ${ }^{c} / \%$ & TON 值 ${ }^{d}$ \\
\hline 1 & & 99.5 & 95.2 & 182 \\
\hline 2 & & 98.5 & 96.3 & 189 \\
\hline $3^{e}$ & & 99.2 & 96.4 & 190 \\
\hline $4^{f}$ & & 90.6 & 90.0 & 174 \\
\hline
\end{tabular}

${ }^{a}$ Reaction conditions: substrate $(6 \mathrm{mmol})$, catalyst $(5 \mathrm{mg})$, Isopropanol $(5 \mathrm{~mL})$, $\mathrm{H}_{2} \mathrm{O}_{2}(1 \mathrm{~mL}), 50{ }^{\circ} \mathrm{C}$, reaction time: $6 \mathrm{~h} .{ }^{b}$ Conversion for specific products were determined by gas chromatography. ${ }^{c}$ Selectivity $=$ yield/conversion, mol\%. ${ }^{d}$ TON was calculated by the mole number of converted (TON= yield/mole number of total $\mathrm{Cu}$ ). ${ }^{e, f}$ The same conditions, just reaction time is 7 $\mathrm{h}$ and $24 \mathrm{~h}$ respectively.

反应继续进行, 转化率保持不变, 而反应的选择性有所 下降，但仍然保持 $94 \%$ 以上，这也充分说明了氧化反应 的迅速性. 另外我们也探究了高倍双氧水对反应的影 响，如图 $5 b$ 所示，在一定的时间里，把双氧水/底物比值 增加到 20 时, 发现转化率 $100 \%$, 选择性仍然能保持 $80 \%$ 以上，当该比值增加到 50 时，转化率 $100 \%$ ，选择性 下降到 $50 \%$ 以下. 当比值为 20 时，按理论来说，双氧水 远远过量，会导致硫醚键过度氧化生成砜，然而实验发 

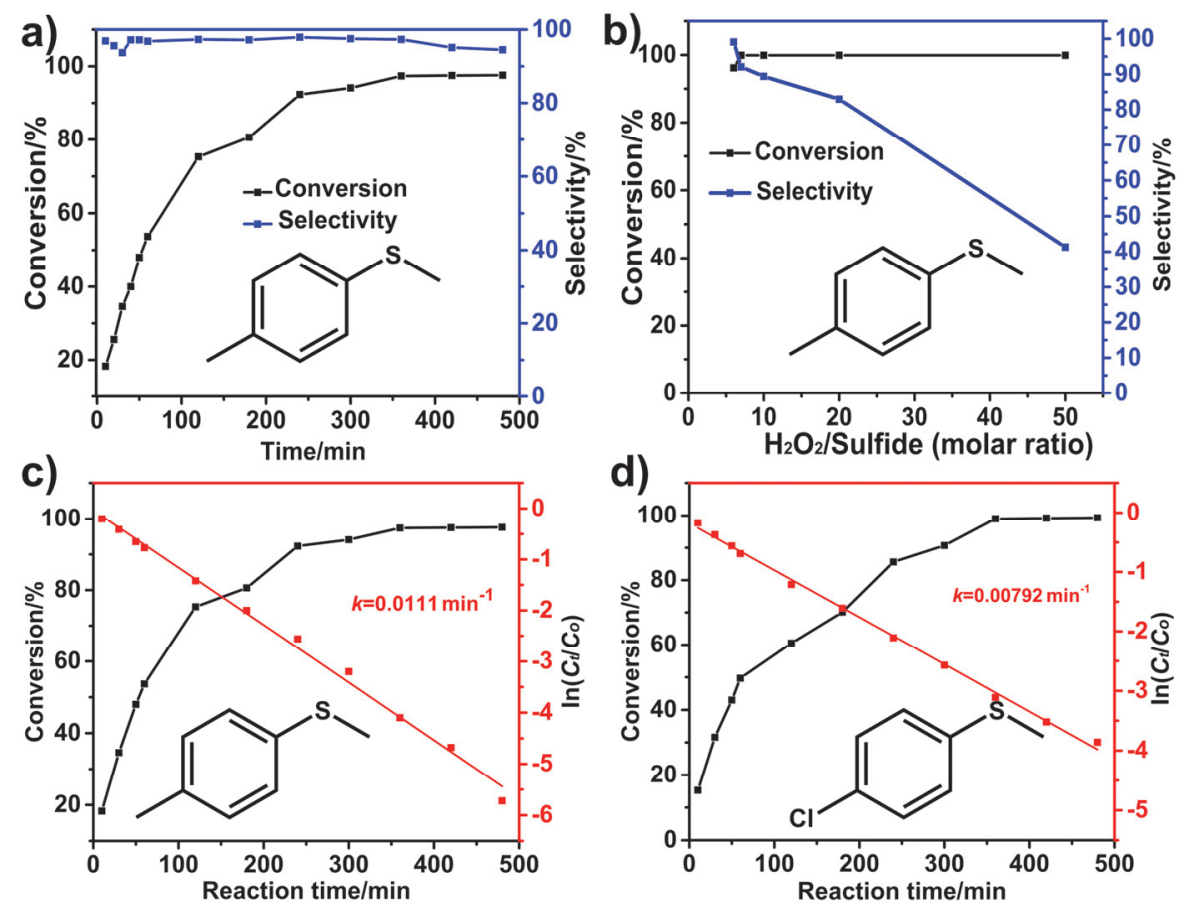

图 5 (a) 时间对氧化反应的影响. (b) 超过量的氧化剂对 4-甲基茴香硫醚氧化的反应的影响. (c) 4-甲基茴香硫醚氧化的转化率和 $\ln \left(C_{t} / C_{0}\right)$ 与时间 的关系图. (d) 4 -氯茴香硫醚氧化的转化率和 $\ln \left(C_{t} / C_{0}\right)$ 与时间的关系图.

Figure 5 (a) Effect of time on oxidation reaction. (b) Effect of oxidant large amount on methy $p$-tolylsulfide oxidation reaction. (c) Methy $p$-tolylsulfide oxidation conversion and $\ln \left(C_{t} / C_{0}\right)$ versus the reaction time. (d) 4-chlorothioanisol oxidation conversion and $\ln \left(C_{t} / C_{0}\right)$ versus the reaction time.

现，除了转化率几乎 $100 \%$ ，仍然能保持很高的选择性， 这与前人很多报道工作不一样, 分析认为, 主要是因为 $\mathrm{Cu}-\mathrm{BDT}$ 的疏水性能迅速将催化氧化得到的亚砜脱附, 使亚砜不能继续进一步氧化成砜, 另外 Cu-BDT 中铜的 价态为正一价, 在氧化反应中不利于促进进一步氧化, 这也导致最终产物为亚砜. 为了验证 Cu-BDT 纳米片在 该催化氧化反应中的优势, 在其他合成条件不变的情况 下, 仅将金属前驱体换为醋酸亚铜, 合成了尺寸均一和 表面光滑的块体 $\mathrm{Cu}$-BDT. 催化结果表明, 两种形貌的 $\mathrm{Cu}-\mathrm{BDT}$ 对硫醚的催化氧化能力相差不大, 可能的原因 是整个催化氧化是在 $\mathrm{Cu}-\mathrm{BDT}$ 的表面进行的. 为了进一 步论证 $\mathrm{Cu}-\mathrm{BDT}$ 在整个催化中起着重要的作用, 在其他 催化条件不变情况下(见表 S3), 不加入 Cu-BDT 催化剂, 整个反应的不同底物转化率低, 选择性差, 这说明了 $\mathrm{Cu}-\mathrm{BDT}$ 不仅对该反应有很好的促进作用且能明显提高 亚砜的选择性.

\subsection{2 反应动力学的研究}

为了进一步理解反应的动力学, 我们控制了催化剂 的量、反应底物的量、氧化剂的量及反应温度, 挑选了 4-甲基茴香硫醚、4-氯茴香硫醚作为反应底物 ${ }^{[30,31]}$. 如 图 $5 \mathrm{c}$ 和 $5 \mathrm{~d}$ 所示, 是转化率、 $\ln \left(C_{t} / C_{0}\right)$ 与时间的关系图, 图中的红点可以拟合成线性的直线, 这说明了 $\mathrm{Cu}-\mathrm{BDT}$ 催化的反应与报道的均相催化体系是一致的, 且都符合

一级反应动力学. 反应动力学常数 $k$ 可以根据以下公式
进行计算:

$$
\ln \left(C_{t} / C_{0}\right)=-k t
$$

这里的 $C_{0}$ 为硫醚类化合物的初始浓度, $C_{t}$ 为 $t$ 时刻的硫 醚类化合物浓度.

根据公式和拟合的直线，可以算出了底物为 4-甲基 茴香硫醚的动力学常数为 $k_{1}=0.0111 \mathrm{~min}^{-1}, 4$-氯基茴香 硫醚的动力学常数为 $k_{2}=0.00792 \mathrm{~min}^{-1}$, 从两个底物的 氧化的动力学常数来看, $\mathrm{Cu}-\mathrm{BDT}$ 可以比较快速地催化 硫醚类化合物氧化成亚砜，且 4-甲基茴香硫梄的动力学 常数大于 4 -氯基茴香硫醚的动力学常数, 说明前者的氧 化速率大于后者的氧化速率，这是因为，整个反应体系 为自由基引发的发应，4-甲基茴香硫醚中甲基为给电子 基团, 增加了硫醚中硫原子的电子云密度, 而 4-氯茴香 硫醚中的氯基为吸电子基团，降低了硫醚中硫原子的电 子云密度，导致 4-氯茴香硫醚的亲核性更弱，从而导致 了氧化速率略小于 4-甲基茴香硫醚的氧化速率. 总而言 之, 硫醚类化合物氧化的效果一定程度上取决于底物的 亲核性，底物亲核性越强，硫醚类化合物氧化效果越好.

\subsection{3 催化剂稳定性测试及材料表征}

为了研究催化剂的使用寿命, 选取了 4-甲基茴香硫 醚为考察对象, 将 $5 \mathrm{mg} \mathrm{Cu}$-BDT 催化剂溶于 $5 \mathrm{~mL}$ 的异 丙醇溶剂中, 加入 $1 \mathrm{mmol}$ 的 4-甲基茴香硫醚, 最后加入 $6 \mathrm{mmol}$ 的双氧水, $50{ }^{\circ} \mathrm{C}$ 反应 $6 \mathrm{~h}$. 每次循环结束后, 将 催化剂用乙醇洗三遍后, 烘干后做下一个循环. 催化循 
环 5 次后发现，如图 6a 所示，转化率由 98\%降至 93\%, 催化剂有一定的失活，选择性由 96\%降至 95\%, 几乎保 持不变, 产率由 $94 \%$ 降至 $88 \%$, 反应的产率有较明显的 下降, 主要的原因可能是多次循环后, 催化剂每次循环 有一定的质量损失. 为了证明催化剂在循环过程没有发 生破坏, 如图 $6 \mathrm{~b}$ 所示, 将循环后的催化剂用 SEM 表征, 与图 $2 \mathrm{a}$ 相比可以看出, 催化剂几乎保持二维片状结构, 只是表面有少许的褶皱. 反应前后的 XRD 和 XPS 分析 表明 5 次循环后的主峰位置几乎保持不变, 说明了 $\mathrm{Cu}-\mathrm{BDT}$ 表现出非常优异的稳定性.

\section{3 结论}

本工作基于自下而上的合成方法，用廉价的 $\mathrm{CuCl}$ 为金属前驱体，1,4-苯二硫醇为有机配体，一系列表征 证明可以简易地合成大量的纳米片层材料(Cu-BDT). 基于 $\mathrm{Cu}-\mathrm{BDT}$ 的疏水特性, 考察了底物的量、催化剂的 量、反应温度及反应时间对氧化反应的影响, 在最佳的 优化条件下, 各类底物的选择性和转化率大于 $90 \%$. 反 应的动力学研究表明, $\mathrm{Cu}-\mathrm{BDT}$ 可以快速地催化硫醚类 化合物氧化成亚砜. 催化循环五次后, Cu-BDT 仍保持 良好的形貌和结构. 该工作为大批量合成二维金属配合 物纳米片提供了新思路, 二维金属有机配合物纳米片因 其丰富可调的表面配位不饱和位点在催化方面具有广 泛的应用前景.
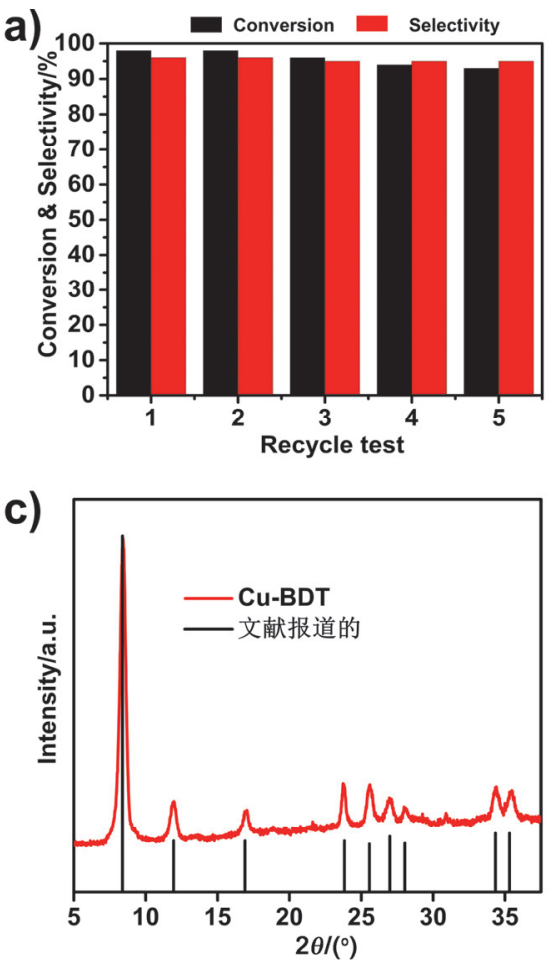

\section{4 实验部分}

\section{1 实验试剂与仪器}

氯化亚铜 $(\mathrm{CuCl},>90 \%)$ 购买于北京普益华科技有 限公司、1,4-苯二硫醇(1,4-Benzenedithiol, 98\%, GC)购买 于北京伊诺凯科技有限公司、乙醇 $(99.5 \%$, 超干，水 $\leqslant$ $50 \mathrm{mg} / \mathrm{L}$ )购买于北京迈瑞达科技有限公司、超干乙腈 (99.9\%, Extra Dry with molecular sieves, Water $\leqslant 50$ $\mathrm{mg} / \mathrm{L})$ 购买于北京伊诺凯科技有限公司、高纯氮气 $(99.9999 \%)$ 购买于北京永圣气体科技有限公司、云母片 $\mathrm{KAl}_{2}\left(\mathrm{AlSi}_{3} \mathrm{O}_{10}\right)(\mathrm{OH})_{2}$ 购买于北京中镜科仪有限公司. 实 验中使用去离子水采用美国 Millipore 公司 Milli-Q (0.22 $\mu \mathrm{m}$ )制备. 茴香硫醚(Thioanisole，99\%)购买于百灵威科 技有限公司、4-甲基茴香硫醚(Methy $p$-tolylsulfide, 99\%) 购买于阿拉丁科技有限公司、4 - 氯茴香硫醚 (4-Chlorothioanisole, 98\%)购买于阿拉丁科技有限公司、 二苯硫醚(Diphenyl sulfide, 98\%)购买于阿拉丁科技有限 公司、双氧水 $\left(\mathrm{H}_{2} \mathrm{O}_{2}, 35 \%\right)$ 和异丙醇(分析纯)购买于北京 化工厂所有试剂均为分析纯，使用前均未经特殊处理. 所得产物的形貌和尺寸均使用日本 Hitachi 公司 S-8220 型扫描电子显微镜(SEM, 工作电压 $6 \mathrm{kV}$ )及美 国 FEI 公司的 Tecnai $\mathrm{G}_{2} 20 \mathrm{ST}$ 透射电子显微镜(TEM，工 作电压 $200 \mathrm{kV}$ )进行表征. 扫描透射电子显微镜 (Scanning Transmission Electron Microscopy, STEM)、高角环
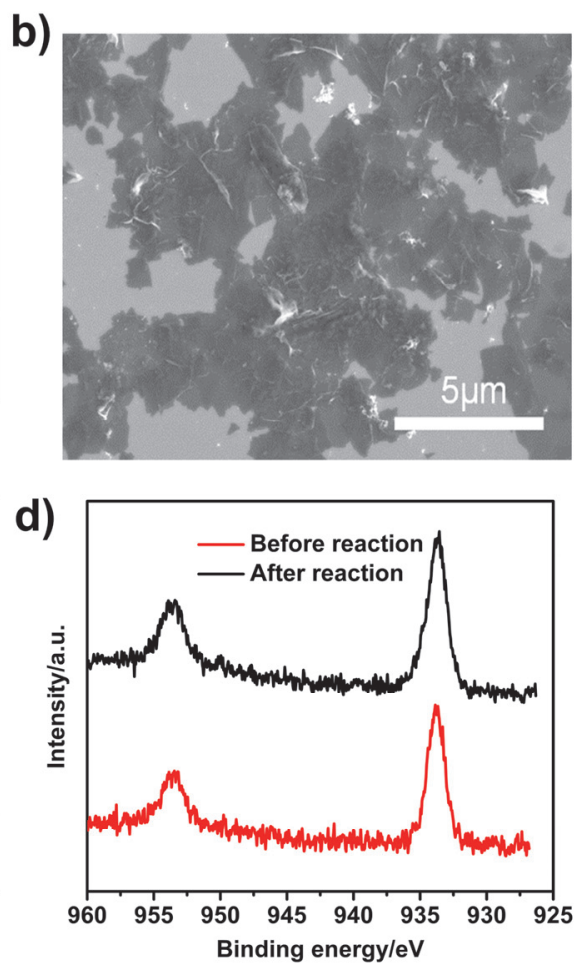

图 6 (a) 催化氧化 4-甲基茴香硫醚的循环测试. (b) 五个催化循环后 Cu-BDT 纳米片的扫描电镜图. (c) Cu-BDT 纳米片反应前后的 XRD. (d) $\mathrm{Cu}-\mathrm{BDT}$ 纳米片反应前后的铜 $2 \mathrm{p}$ 的 XPS 谱图.

Figure 6 (a) The recycle test of methy $p$-tolylsulfide oxidation. (b) SEM image of Cu-BDT nanosheets after 5 recyclable tests. (c) The XRD of Cu-BDT nanosheets before and after reaction. (d) XPS spectra of $\mathrm{Cu} 2 \mathrm{p}$ before and after reaction. 
形暗场照片(HAAHP-STEM)和能量散射 $X$ 射线元素分 析(Energy Dispersive X-ray Spectroscopy Mapping)在美 国 FEI 公司的配置高角环形暗场探测器 (High-Angle Annular Dark Field (HAADF) Detector) 的 G2-F20 U-TWIN 型透射电子显微镜(工作电压 $200 \mathrm{kV}$ )上完成. 所得样品的物相表征通过使用 D/MAX-TTRIII (CBO)型 多晶 $\mathrm{X}$ 射线衍射仪(使用 $\mathrm{Cu}$ 靶 $\mathrm{K} \alpha$ 射线, $\lambda=0.15418 \mathrm{~nm}$, 工作电流 $300 \mathrm{~mA}$, 工作电压 $50 \mathrm{kV}$ )来完成. 样品的傅 里叶变换红外光谱 (Fourier transform infrared, FT-IR)使 用美国 PerkinElmer 公司 PE2000 红外光谱仪, 采用 $\mathrm{KBr}$ 压片法完成. 样品的激光拉曼光谱(Renishaw, Raman)使用英国 Renishaw 公司的 Renishaw in Via plus 激 光拉曼光谱仪, 采用压片机完成. 样品的亲疏水性使用 德国制造的 DSA100 接触角测试仪, 采用压片机压片完 成. 样品的分离是在长沙湘仪离心机仪器有限公司 H-1650 型 $50 \mathrm{~mL}$ 离心机和 Eppendorf 公司 Centrifuge 5424 型 $10 \mathrm{~mL}$ 离心机上进行. Cu-BDT 的性能评价在 磁力搅拌高压反应釜(福州克雷斯试验设备有限公司) 装置中进行, 保护气体为高纯氮气( $99.999 \%)$. 反应产物 定性分析采用气相色谱一质谱联用仪(Thermo DSQ, 色 谱柱: TR-5MS) 完成, 定量分析采用岛津气相色谱仪 (GC-2010 Plus, 色谱柱: Rtx-5 $30 \mathrm{~m} \times 0.25 \mathrm{~mm} \times 0.25$ $\mu \mathrm{m})$, 相应的 GC 图表征见图 S1 S S4.

\subsection{Cu-BDT 的合成方法}

首先将 $100 \mathrm{mg} \mathrm{CuCl}(1 \mathrm{mmol})$ 溶于 $20 \mathrm{~mL}$ 的超干乙 腈溶液中, 然后将 $142 \mathrm{mg}$ 1,4-苯二硫醇 $(1 \mathrm{mmol}$ )溶于 20 $\mathrm{mL}$ 的超干乙腈中, 上述两种溶液置于超声机中超声 10 $\mathrm{min}$, 保证完全溶解. 将 $20 \mathrm{~mL}$ 的 1,4-苯二硫醇溶液倒入 $100 \mathrm{~mL}$ 的两口瓶中, 抽真空置换成氮气保护, 此过程重 复三遍, 以保证两口瓶内的氧气被充分排净, 然后将反 应瓶置于带有搅拌的冰浴中, 在剧烈的搅拌下, 用注射 器慢慢滴加 $\mathrm{CuCl}$ 溶液, 完毕之后, 在搅拌的冰浴中保 留 $30 \mathrm{~min}$ 后, 然后将冰浴锅移走, 把带有氮气保护的两 口瓶置于室温中, 静置 $72 \mathrm{~h}$, 反应完毕. 所得产物用离 心机 $10000 \mathrm{r} / \mathrm{min}$ 离心 $10 \mathrm{~min}$, 所得沉淀用超干乙腈洗 涤两次, 然后用超干乙醇洗涤两次, 将所得固体放入 $60{ }^{\circ} \mathrm{C}$ 真空干燥箱中干燥 $12 \mathrm{~h}$, 即可得黄色固体, 将该 材料命名为 $\mathrm{Cu}-\mathrm{BDT}$.

\section{3 催化反应的评价}

为了排除空气对反应的干扰, 我们采用磁力搅拌高 压反应釜作为反应器. 首先将 $5 \mathrm{mg} \mathrm{Cu}$-BDT 溶于 $5 \mathrm{~mL}$ 异丙醇中, 然后加入 $1 \mathrm{mmol}$ 底物, 超声至催化剂均匀 分散后，将该反应液移至高压反应釜中，加入一定量的 双氧水, 氮气置换三次后, $50{ }^{\circ} \mathrm{C}$ 搅拌一定时间. 反应结 束后催化剂经离心分离, 然后催化剂用乙醇洗涤三次烘 干用于其他表征.

\section{References}

[1] Cao, F.-F.; Zhao, M.-T.; Yu, Y.-F.; Chen, B.; Huang, Y.; Yang, J.; Cao, X.-H.; Lu, Q.-P.; Zhang, X.; Zhang, Z.-C.; Tan, C.-L.; Zhang, H. J. Am. Chem. Soc. 2016, 138, 6924.

[2] El-Kady, M. F.; Veronica, S.; Sergey, D.; Richard, B. K. Science 2012, 335, 1326.

[3] Wu, C.-Z.; Lu, X.-L.; Peng, L.-L.; Xu, K.; Peng, X.; Huang, J.-L.; Yu, G.-H.; Xie, Y. Nat. Comm. 2013, 4, 3431.

[4] Yang, X.-W.; Cheng, C.; Wang, Y.-F.; Qiu, L.; Li, D. Science 2013, 341,6145 .

[5] Huang, W.; Li, Y.-G. Chinese J. Chem. 2019, 37, 12.

[6] Wang, H.-T.; Yuan, H.-T.; Seung, S. H.; Li, Y.-B.; Cui, Y. Chem. Soc. Rev. 2015, 44, 2664.

[7] Deng, D.; Novoselov, K. S.; Fu, Q.; Zheng, N.-F.; Tian, Z.-Q.; Bao, X.-H. Nat. Nanotech. 2016, 11, 218.

[8] Song, F.; Hu, X.-L. J. Am. Chem. Soc. 2018, 136, 16481.

[9] Song, F.; Hu, X.-L. Nat. Comm. 2014, 5, 5447.

[10] Zheng, Y.; Yan, J.; Lei, G.; Mietek, J.; Qiao, S.-Z. Angew. Chem., Int. Ed. 2013, 125, 3192.

[11] Yu, J.; Yang, Y.-S.; Wei, M. Acta Chim. Sinica 2019, 77, 1129 (in Chinese). (余俊, 杨宇森, 卫敏, 化学学报, 2019, 77, 1129.)

[12] Jiao, C.-L.; Wang, W.; Liu, J.; Yuan, Y.-X.; Xu, M.-M.; Yao, J.-L. Acta Chim. Sinica 2018, 76, 526 (in Chinese). (焦岑蕾, 王炜, 刘 娇, 袁亚仙, 徐敏敏, 姚建林, 化学学报, 2018, 76, 526.)

[13] Naguib, M.; Gogotsi, Y. Acc. Chem. Res. 2014, 48, 128.

[14] Niu, L.-Y.; Coleman, J. N.; Zhang, H.; Shin, H.; Chhowalla, M.; Zheng, Z.-J. Small 2016, 12, 272.

[15] Stankovich, S.; Dikin, D. A.; Dommett, G. H. B.; Kohlhaas, K. M.; Zimney, E. J.; Stach, E. A.; Piner, R. D.; Nguyen, S. T.; Ruoff, R. S. Nature 2006, 442, 282.

[16] Sakamoto, J.; Jeroen, v-H.; Lukin, O.; Schlüter, A.-D. Angew. Chem., Int. Ed. 2009, 48, 1030.

[17] Huang, X.; Zeng, Z.-Y.; Zhang, H. Chem. Soc. Rev. 2013, 42, 1934.

[18] Chu, W.-Y.; Tang, X.; Li, Z.; Lin, J.-C.; Qian, J.-S. Acta Chim. Sinica 2018, 76, 549 (in Chinese). (楚婉怡, 唐笑, 李振, 林景诚, 钱 觉时, 化学学报, 2018, 76, 549.)

[19] Sakamoto, R.; Takada, K.; Pal, T.; Maeda, H.; Kambecd, T.; Nishiharaa, H. Chem. Coummn. 2017, 53, 5781.

[20] Dong, R.-H.; Pfeffermann, M.; Liang, H.-W.; Zheng, Z.-K.; Zhu, X.; Zhang, J.; Feng, X.-L. Angew. Chem., Int. Ed. 2015, 54, 12058.

[21] Kambe, T.; Sakamoto, R.; Hoshiko, K.; Takada, K.; Miyachi, M.; Ryu, J.-H.; Sasak, S.; Kim, J.; Nakazato.; Takat, M.; Nishihara, H. J. Am. Chem. Soc. 2013, 135, 2462.

[22] Clough, A. J.; Yoo, J. W.; Mecklenburg, M. H.; Marinescu, S. C. J. Am. Chem. Soc. 2014, 137, 118.

[23] Maeda, H.; Sakamoto, R.; Nishihara, H. Langmuir 2016, 32, 2527.

[24] Sugimori, A.; Akiyama, T.; Kajitani, M.; Sugiyama, T. B. Chem. Soc. Jpn. 1999, 72, 879.

[25] Dong, R.-H.; Pfeffermann, M.; Liang, H.-W.; Zheng, Z.-K; Zhu, X.; Zhang, J.; Feng, X.-L. Angew. Chem., Int. -Ed. 2015, 54, 12058.

[26] Huang, X.; Sheng, P.; Tu, Z.-Y.; Zhang, F.-J.; Wang, J. H.; Geng, H.; Zou, Y.; Di, C.-A.; Yi, Y.-P.; Sun, Y.-M.; Xu, W.; Zhu, D.-B. Nat. Comm. 2015, 6, 7408 .

[27] Huang, X.; Li, H.-S.; Tu, Z.-Y.; Liu, L.-Y.; Wu, X.-Y.; Chen, J.; Liang, Y.-Y.; Zou, Y.; Yi, Y.-P.; Zhu, D.-B. J. Am. Chem. Soc. 2018, $140,15153$.

[28] Takada, K.; Sakamoto, R.; Yi, S.-T.; Katagiri, S.; Kambe, T.; Nishihara, H. J. Am. Chem. Soc. 2015, 137, 4681.

[29] Xu, G.; Otsubo, K.; Yamada, T.; Sakaida, S.; Kitagawa, H. J. Am. Chem. Soc. 2013, 135, 7438.

[30] Bauer, T.; Zheng, Z.-K.; Renn, A.; Enning, R.; Stemmer, A.; Sakamoto, J.; Schlüter, A. D. J. Am. Chem. Soc. 2011, 123, 8025.

[31] Yang, R.-T.; Maldonado, A. J.; Yang, F.-H. Science 2003, 301, 79.

[32] Li, T.-F.; Zhang, W.; Chen, W.; Miras, H. N.; Song, Y.-F. ChemCatChem 2017, 10, 188.

[33] Doherty, S.; Knight, J. G.; Carroll, M. A.; Ellison, J. R.; Hobson, S. J.; Stevens, S.; Hardacre, C.; Goodrichb, P. Green Chem. 2015, 17, 1559.

[34] Nisar, A.; Zhuang, J.; Wang, X. Adv. Mater. 2011, 23, 1130.

[35] Nisar, A.; Lu, Y.; Zhuang, J.; Wang, X. Angew. Chem., Int. Ed. 2011, 123,3245 .

[36] Schrauzer, G.; Prakash, H. Inorg. Chem. 1975, 14, 1200.

[37] Hu, C.; Hu, C.-Y.; Ma, Q.-Y.; Hung, S.-F.; Chen, Z.-N.; Ou, D.-H.; Ren, B.; Chen, C.-M.; Fu, G.; Zheng, N.-F. Chem 2017, 3, 122. 
[38] Gaarenstroom, S.; Winograd, N. J. Chem. Phys. 1977, 67, 3500.

[39] Rupp, H.; Weser, U. BBA-Protein Structure 1976, 446, 151

[40] Poulston, S.; Parlett, P. M.; Stone, P.; Bowker, M. Surf. Interface. Anal. 1996, 24, 811.

[41] Zhang, Z.; Long, J.-L.; Yang, L.-F.; Chen, W.-K. Chem. Sci. 2011, 2, 1826.

[42] Sandhyarani, N.; Pradeep, T. J. Mater. Chem. 2001, 11, 1294.

[43] Xia, C.; Xia, Y.; Zhu, P.; Fan, L.; Wang, H.-T. Science 2019, 366,
226.

[44] Guo, X.-K.; Xu, M.-X.; She, M.-Y.; Zhu, Y.; Shi, T.-T.; Chen, Z.-X.; Peng, L.-M.; Guo, X.-F.; Lin, M.; Ding, W.-P. Angew. Chem., Int. Ed. 2020, 59, 2606

[45] Tan, B.; Wu, W.-H.; Liu, X.; Zhang, Y.-B.; Quan, X.; Zhao, H.-M. Nanoscale 2017, 9, 18699.

[46] Xiao, F.-S.; Sun, J.-M.; Meng, X.-J.; Yu, R.-B.; Yuan, H.-M.; Xu, J.-N.; Song, T.-Y.; Jiang, D.-Z.; Xu, R.-R. J. Catal. 2001, 199, 273.

(Cheng, B.) 